\title{
Geometric Laws of Vortex Quantum Tunneling
}

\author{
Uwe R. Fischer ${ }^{1,2}$ 因 \\ ${ }^{1}$ Institut für Theoretische Astrophysik, Universität Tübingen, Auf der Morgenstelle 10, D-72076 Tübingen \\ ${ }^{2}$ Low Temperature Laboratory, Helsinki University of Technology, P.O. Box 2200, FIN-02015 HUT
}

(October 22, 2017)

\begin{abstract}
In the semiclassical domain the exponent of vortex quantum tunneling is dominated by a volume which is associated with the path the vortex line traces out during its escape from the metastable well. We explicitly show the influence of geometrical quantities on this volume by describing point vortex motion in the presence of an ellipse. It is argued that for the semiclassical description to hold the introduction of an additional geometric constraint, the distance of closest approach, is required. This constraint implies that the semiclassical description of vortex nucleation by tunneling at a boundary is in general not possible. Geometry dependence of the tunneling volume provides a means to verify experimental observation of vortex quantum tunneling in the superfluid Helium II.
\end{abstract}

PACS numbers: 66.35.+a, 67.40.Vs

The question how a singular line with circulating flow around it enters a fluid in which friction is absent has pervaded the theory of superfluids for decades. It is currently believed that at the absolute zero of temperature, a quantized vortex can come into the superfluid by a tunneling process. In this process, Galilean invariance has to be broken because there exists no absolute reference frame in the homogenous bulk if the temperature equals zero. The formalism able to describe the quantum mechanical process of tunneling depends sensitively on the relevant length scales involved. If the scale after tunneling, when the vortex is able to move freely in the superfluid, is much larger than the core size of the vortex, we are able to resort to a hydrodynamic, i.e. semiclassical description 11. If this scale is near the core size $\xi$, a description of vortex tunneling requires knowledge of the dynamic many-body structure of the vortex core, a formidable goal yet to be achieved. In this paper, we address the question of geometric laws involved in the exponent of vortex quantum tunneling in the semiclassical approximation and investigate the limits imposed on this exponent by the shape of the flow obstacle breaking Galilean invariance. We show that the usual description of tunneling with a vortex moving in a pinning potential generated by some flow obstacle is limited by a geometric constraint related to the vortex core size.

The tunneling rate of the vortex is proportional to $\exp \left[-S_{e}(E) / \hbar\right]$, where $S_{e}(E) \gg \hbar$ is the Euclidean action as a function of vortex energy $E$ [2]:

$$
S_{e}(E)=\oint d \sigma \oint d \boldsymbol{K} \cdot \boldsymbol{P} .
$$

Here, $\boldsymbol{K} \equiv-i \boldsymbol{X}(\sigma)$ is the imaginary collective vortex coordinate and $\boldsymbol{P}(\sigma)$ the local vortex momentum per unit $\sigma$-length (with $\sigma$ the arc length parameter labeling points on the vortex string). The integral has to be performed along the classical vortex path with constant energy. We assume dissipation to be negligible in the very pure sys- tem under consideration. The only driving mechanism behind the tunneling transition we consider is the superfluid Magnus force at zero temperature.

We first show in its full generality that the action (1) in an incompressible fluid is a geometric quantity. The corrections induced by the mass of the vortex have been shown to be negligibly small in the semiclassical limit [3. 5 . They will consequently be neglected here.

In analogy to electrodynamics [6], the canonical momentum $\boldsymbol{P}$ in an incompressible superfluid corresponds to the vector potential and is a gauge dependent vector with components

$$
P_{i}=\frac{N_{v}}{N_{s}} h \rho_{0} b_{i j} X^{\prime j},
$$

where $h \rho_{0}$ is the bulk particle number density multiplied by Planck's action quantum $h, X^{\prime j} \equiv \partial X^{j} / \partial \sigma$ the line tangent. We took the quantum of circulation to be $\kappa=\left(N_{v} / N_{s}\right) h / m$, with $N_{v}$ the winding number of the vortex and $N_{s}$ the number of real particles in the superfluid boson (a Cooper pair has $N_{s}=2$ ). The antisymmetric gauge tensor $b_{\mu \nu}$ of string dynamics [7], a $3+1 d$ generalization of the stream function of classical hydrodynamics, is defined by the dual transformation $v^{\gamma} \epsilon_{\gamma \alpha \mu \nu}=b_{\mu \nu, \alpha}+b_{\alpha \mu, \nu}+b_{\nu \alpha, \mu}$ from the conservation of the four-current $J^{\mu}=\rho v^{\mu}$ (with $\rho=\rho_{0}$ ). Let $\boldsymbol{e}_{1}, \boldsymbol{e}_{2}, \boldsymbol{e}_{\sigma} \equiv \boldsymbol{X}^{\prime}$ be a righthanded co-ordinate basis on the string. In our non-relativistic case, we have $-\oint \oint P_{a} d X^{a} \wedge d \sigma=\left(N_{v} / N_{s}\right) h \rho_{0} \iiint \sqrt{g} d X^{1} \wedge d X^{2} \wedge d \sigma$, where $g$ is the determinant of an arbitrary metric in the basis $\boldsymbol{e}_{a}, \boldsymbol{e}_{\sigma}(a=1,2)$. This yields for the components of $\boldsymbol{P}$ in the directions $\boldsymbol{e}_{1}, \boldsymbol{e}_{2}$ the relation

$$
\partial_{2} P_{1}-\partial_{1} P_{2}=\frac{N_{v}}{N_{s}} h \rho_{0} \sqrt{g} .
$$

Equation (3) represents the conjugateness of positions and momenta in the directions $\boldsymbol{e}_{1}, \boldsymbol{e}_{2}$ in its general form. As a well-known example, if we gauge $P_{2}=0$, then, in 
Cartesian co-ordinates, $P_{X}=\left(N_{v} / N_{s}\right) h \rho_{0} Y$, the gauge to be used below in the application to the $2 \mathrm{~d}$ case. We are now able to rewrite the action (11) as

$$
\frac{S_{e}(E)}{h}=\frac{N_{v}}{N_{s}} \rho_{0} \iiint \sqrt{g} d \sigma d Z^{1} d Z^{2} .
$$

The new co-ordinate differentials are $d Z^{1}=\cos \alpha d K^{1}+$ $\sin \alpha d K^{2}, d Z^{2}=-\sin \alpha d X^{1}+\cos \alpha d X^{2}$. The angle $\alpha(\sigma)$ parameterizes the local (co-ordinate) gauge freedom for the momentum of rotations about $\boldsymbol{e}_{\sigma}$, contained in (3). In what follows, $N_{v} / N_{s} \equiv 1$ for simplicity. The gauge invariant expression (4) tells us that the dimensionless action is the number density times the volume which is involved in the tunneling process. Note that the action is scaled with $h$, not $\hbar=h / 2 \pi$. This Volume Law is the dominant contribution to the tunneling exponent in a superfluid in which the non-relativistic limit is the physically relevant one. The number of particles in the effective tunneling volume plays the role of the quantum number in a Bohr-Sommerfeld type quantization of (1). We stress that this result holds however complicated the motion of the vortex string actually is as long as we are in the semiclassical domain.

What is of interest to us here are the limits which govern the actual value of the tunneling volume for a given external geometry. To tackle the general features of the problem, we restrict ourselves to the case of point vortices in $2 \mathrm{~d}$, where we can discard the complicated $\sigma$ dependence in (4). We are then in the position to use the tools of conformal transformation to calculate the energy of the vortex as a function of its coordinates near some irregular boundary of the superfluid [8]. The simplest realistic geometry of interest to us for this purpose is that of a half-ellipse whose small semi-axis $a$ is in the direction of the flow parallel to the boundary with a much bigger semi-axis $b \gg a$ perpendicular to it. This provides a realistic pinning potential directly related to geometrical properties of the pinning site in contrast to a harmonic shape of the potential with fitting parameters used, e.g., in 9, 4 .

We can map the readily solvable problem of a vortex in the presence of a half-circle by the inverse of the Joukowski transformation [8] to that of the half-ellipsevortex geometry in question. The vortex energy contains three terms. The first term is half the self energy of a vortex pair and the second, which gives the pinning potential, stems from the two image vortices inside the circle respectively ellipse required to satisfy the boundary conditions. The last one comes from a superimposed external flow velocity $u$ at infinity in the negative $x$ direction parallel to the boundary. We use appropriate elliptic co-ordinates defined by $x=l \sinh \chi \cos \eta$, $y=l \cosh \chi \sin \eta$, in which choosing $\chi=\chi_{0}$ gives us $a=l \sinh \chi_{0}, b=l \cosh \chi_{0}$, with $l=\sqrt{b^{2}-a^{2}} \approx b \gg \xi$ the overall length scale. If we normalize the energy as a function of vortex position $\chi_{1}, \eta_{1}$ by $\tilde{E} \equiv 4 \pi E / m \rho_{0} \kappa^{2}$, it takes the form

$$
\begin{array}{r}
\tilde{E}=\ln \left[\frac{a+b}{\xi} \frac{\exp \left(\chi_{1}-\chi_{0}\right)\left|\sin \eta_{1}\right| \sinh \left(\chi_{1}-\chi_{0}\right)}{\left(\sinh ^{2}\left(\chi_{1}-\chi_{0}\right)+\sin ^{2} \eta_{1}\right)^{1 / 2}}\right] \\
-\frac{4 \pi u(a+b)}{\kappa} \sinh \left(\chi_{1}-\chi_{0}\right)\left|\sin \eta_{1}\right| .
\end{array}
$$

We cannot solve for the path of constant $\tilde{E}$ analytically if we allow for any values of $\xi, a, b$ and $u$ in the semiclassical domain. What we want to show here are general features with respect to the geometrical quantities involved in the tunneling exponent. Hence for the velocity $u$ we consider the limit $u \ll \kappa / 2 \pi l \ll v_{L}$, where $v_{L} \equiv \kappa / 2 \pi \xi$ is the characteristic velocity associated with the manybody quantum structure of the fluid ('Landau' velocity). The external current is limited by $2 u / v_{L} \ll a / b$, such that the velocity without vortex at the ellipse top remains well below the critical $v_{L}$.

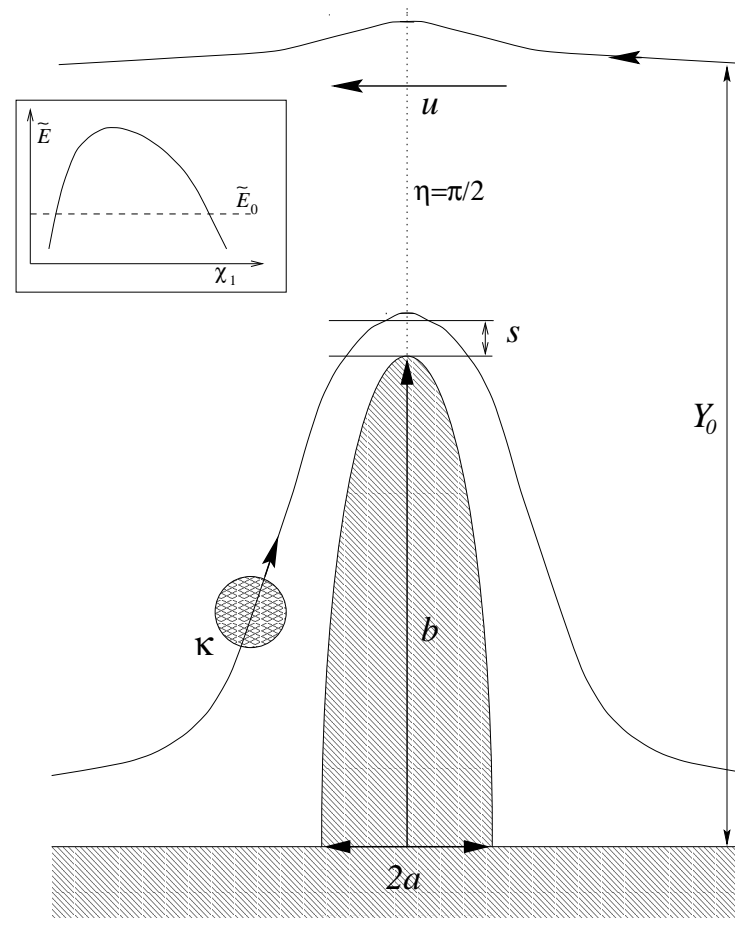

FIG. 1. The two vortex paths of the same constant energy $\tilde{E}_{0}$. The vortex on the path passing the ellipse top on a distance slightly larger than $s \geq \xi$ has to tunnel to the second path, which is at a distance $\simeq Y_{0}$ from the boundary. The small inset shows the shape of the barrier from (5) for $\eta=\eta_{1}=\pi / 2$.

We now come to the crucial point of our argumentation: We require that the distance of the vortex to the ellipse top, the point of closest approach of the vortex, is slightly more than $\xi$, because any smaller distance to the boundary would invalidate a description of the vortex 
in semiclassical terms. This requirement can be imposed by $\delta \chi\left(\eta_{1}=\pi / 2\right)=s / a$, with $\xi \leq s \ll a$, and where we set $\delta \chi \equiv \chi_{1}-\chi_{0}$. The energy $\overline{\tilde{E}} \equiv \tilde{E}_{0}$ of the paths we consider (see Fig. 11) is then fixed to be

$$
\tilde{E}_{0} \equiv \tilde{E}(a, b, s, \xi, u)=\ln \left[\frac{a+b}{a} \frac{s}{\xi}\right]-\frac{4 \pi u(a+b)}{\kappa} \frac{s}{a} .
$$

For low velocity, we can write $\exp \tilde{E}_{0} \simeq(b / a)(s / \xi)$, the value to be used in the following.

There are two vortex paths having the same energy (Fig. 1). One of these is that of the vortex at the boundary and closely following the ellipse, for which $\delta \chi \ll 1$. We obtain from (5), if $\sin \eta_{1} \gg \sinh \delta \chi, b / a \gg 1$ :

$$
Y_{\mathrm{E}} \simeq \frac{\kappa}{4 \pi u} \frac{1}{\delta \chi} \ln \left[\frac{a}{s} \delta \chi\right]
$$

The other vortex path of constant energy $Y_{N}\left(\chi_{1}, \eta_{1}\right)$ is located far away from the ellipse and the boundary. The vortex has to tunnel through the barrier represented by (5) from the ellipse path (7) to this trajectory. In lowest order of the velocity $u$, this path follows a constant distance $Y_{0}$ to the boundary:

$$
Y_{\mathrm{N}} \simeq Y_{0} \equiv \frac{\kappa}{4 \pi u} \ln \left(\frac{\kappa}{2 \pi u} \frac{a / b}{s}\right) .
$$

The two paths (8) and (7) do never meet provided that $2 u / v_{L} \ll a / b$ holds. To describe the tunneling trajectory of constant energy, we have to use complex co-ordinates in the $Z^{1}, Z^{2}$ space contained in (4) such that they meet at some point in this space. We can then obtain a closed escape path. To the end of finding the path, we return to the original Cartesian co-ordinates and write the vortex trajectory in the complex $(K=-i X, Y)$ space as

$$
Y_{\mathrm{E}}^{2}=l^{2} \cosh ^{2}\left(\chi_{0}+\delta \chi\right)+\tanh ^{-2}\left(\chi_{0}+\delta \chi\right) K^{2},
$$

where the deviation from the original ellipse $\delta \chi$ is in general via (7) itself a function of $K, Y$. In lowest order of $\delta \chi / \chi_{0}$, however, (17) yields approximately a constant $\delta \chi \simeq s / a$ equal to its value at the ellipse top. The two trajectories (8) and (9) then cross at

$$
\left|K_{m}\right| \simeq\left(\frac{a}{b}+\frac{s}{a}\right) Y_{0} \equiv \beta Y_{0}
$$

Evaluating the integral (4) leads to the tunneling action (see also Fig. 2):

$$
\begin{aligned}
\frac{S_{e}}{h} & =2 \rho_{0} \int_{0}^{K_{m}}\left(Y_{\mathrm{N}}-Y_{\mathrm{E}}\right) d K \simeq \rho_{0} \beta Y_{0}^{2} \\
& =\frac{1}{16 \pi^{2}} \rho_{0}\left(\frac{a}{b}+\frac{s}{a}\right)\left(\frac{\kappa}{u} \ln \left[\frac{\kappa}{2 \pi u} \frac{a / b}{s}\right]\right)^{2} .
\end{aligned}
$$

We remind the reader that the result in this simple analytical form is valid to lowest order in the small quantities
$2 u / v_{L} \ll a / b \ll 1, s / a \ll a / b$. But the feature that $s, a, b$ enter the action does also hold for more general values in the parameter space. We conclude that the semiclassical action depends on three geometrical quantities: The characteristic lengths parallel and perpendicular to the flow $a, b$ and the closest approach distance $s$ to the ellipse. The quantity $s$ enters because we imposed the condition that the vortex must not get nearer to the surface than $\xi \leq s$. This resulted in an effective rescaling $\xi \rightarrow \xi \exp \tilde{E}_{0}$ of the ultraviolet cut-off in the vortex energy logarithm and a restriction on the effective sharpness $\beta \geq a / b+\xi / a$ of the ellipse.

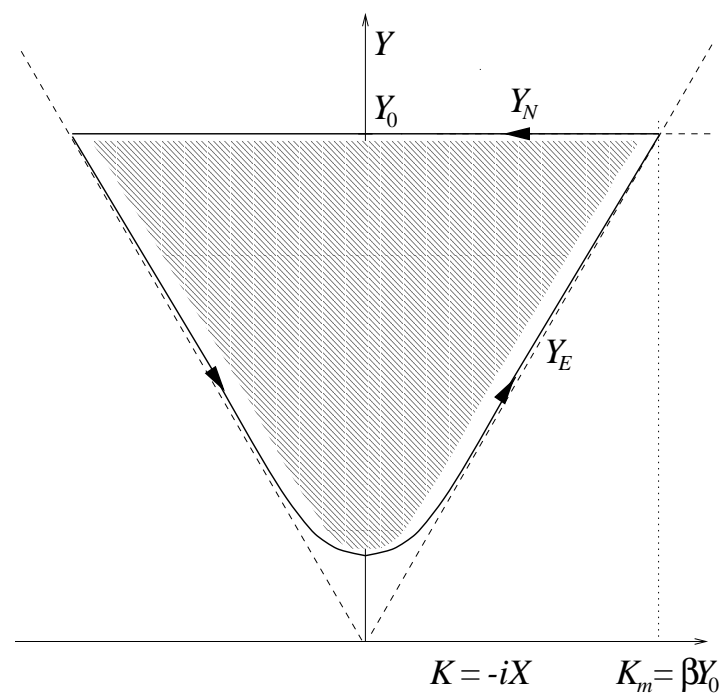

FIG. 2. The area $\simeq \beta Y_{0}^{2}=V_{N}^{(2)}$ enclosed by the vortex path in complex space gives the action (11) in two space dimensions. This area has a lower semiclassical limit $(a / b+\xi / a) Y_{0}^{2}$.

As a generalization of (11), the semiclassical action for a massless vortex in $d$ dimensions will take the form

$$
\frac{S_{e}}{h}=\rho_{0} \beta V_{N}^{(d)}
$$

where $\beta \ll 1$ characterizes the effective dimension of the vortex escape path, i.e. the relative degree to which it is confined to $n$ dimensions by the presence of an asperity which is effectively $n$-dimensional. The quantity $\beta$ is bounded from below by the requirement of semiclassicality for the vortex path at the boundary, as expounded above for the two-dimensional case. The tunneling volume $V_{N}^{(d)}$ is that for a vortex escape path of $\mathrm{O}(d-1)$ symmetry 10, which is the highest possible symmetry if one preferred direction, namely that of the external current, is given. In our $d=2$ case of (11), we have $n=1, \beta \simeq a / b+s / a$ and $V_{N}^{(2)}=Y_{0}^{2}$. In three dimensions $(d=3)$ we have $V_{N}^{(3)}=(4 \pi / 3) R_{0}^{3}$ for a half-ring with radius $R_{0}$.

The conclusions we have drawn should be able to shed light on the question if in experiments on the flow of 
superfluid ${ }^{4} \mathrm{He}$ through sub-micron orifices we are dealing with the quantum tunneling of vortices below about $T=150 \mathrm{mK} 12,13$. Geometry dependence provides a tool to verify that the critical velocity plateau observed there comes indeed from a quantum process and not an alternative process of classical instability, e.g. of the Kadomtsev-Petviashvili type [14], is taking place. In ${ }^{4} \mathrm{He}$, the velocity $v_{L}$ is quite large due to the small coherence length in the order of the interparticle spacing (it equals the Landau velocity $\approx 59 \mathrm{~m} / \mathrm{sec}$ at $p=1$ atm if $\xi \simeq 2.7 \AA$ is taken). The observed critical velocities of flow through the orifice on the temperature independent plateau are of the order of $10 \mathrm{~m} / \mathrm{sec} 13,15$. This leads to materialization radii $R_{0}$ of the order of nanometers $\left(R_{0}\right.$ corresponds to the $2 \mathrm{~d}$ quantity $Y_{0}$ in (8)). A hydrodynamic treatment appears thus justified and there should be a strong dependence of critical velocities on the surface structure of the orifices. The simple model of vortex half rings situated at plane walls with their momentum axis antiparallel to the flow 13,15] does not account for the required explicit incorporation of Galilean invariance violation. The enlargement of the core size at boundaries used in this model to explain observed critical velocities is naturally contained here. It is represented by the condition that a vortex moving in a pinning potential caused by a nontrivial geometry only exists as a semiclassical vortex able to tunnel through the barrier if its distance to the wall is everywhere bigger than $\xi$. This results in a nonzero tunneling energy $\tilde{E}_{0}>0$ of the vortex interpretable as an enlargement of $\xi \rightarrow \xi \exp \tilde{E}_{0}$.

The necessity of nonzero tunneling energy is responsible for the fact that we do not deal with the nucleation of vortices here, i.e. their creation from 'nothing'. The vortex already has to exist at the boundary with a small but nonzero energy such that it can tunnel to get a free vortex. Hence we are dealing with a depinning rather than a nucleation event. In the semiclassical limit the description of the nucleation of zero energy vortices is in general only possible if the curvature radius of the flow obstacle is constant, that is in the case of $d$-1-dimensional spheres. If the curvature radius of the obstacle varies, the distance of the zero energy vortex to the boundary varies and can get smaller than $\xi$ at the point of closest approach, thus violating the requirements of the semiclassical approach.

To conclude, we emphasize again the important fact that violation of Galilean invariance in the direction of the flow is a necessary condition for vortex quantum tunneling to happen at the absolute zero of temperature. The tunneling exponent will depend on geometrical properties of the vortex path associated with curvature scales of the asperities breaking the invariance. That the semiclassical description be valid introduces the additional length scale $s$, the closest approach distance to the flow obstacle. The semiclassical theory of vortex quantum tunneling in a pure superfluid is essentially a geometric theory.
I am indebted to Grisha Volovik for stimulating discussions and helpful comments. This work was supported by the Human Capital and Mobility Programme of the European Union (contract number CHGE-CT94-0069).

* Electronic address: fischer@tat.physik.uni-tuebingen.de

[1] G. E. Volovik, JETP Lett. 15, 81 (1972)

[2] L. D. Landau, E. M. Lifshitz: Quantum Mechanics, Pergamon Press, Second Edition 1965

[3] G. E. Volovik, JETP Lett. 65, 201 (1997)

[4] M. J. Stephen, Phys. Rev. Lett. 72, 1534 (1994)

[5] M. V. Feigel'man, V. B. Geshkenbein, A. I. Larkin, S. Levit, JETP Lett. 57, 711 (1993)

[6] U. R. Fischer, cond-mat/9708074

[7] M. Kalb, P. Ramond, Phys. Rev. D 9, 2273 (1974)

[8] L. M. Milne-Thomson: Theoretical Hydrodynamics, Macmillan, Fifth Edition 1968

[9] P. Ao, D. J. Thouless, Phys. Rev. Lett. 72, 132 (1994)

[10] It has already been pointed out in [5] that we do not deal with the $\mathrm{O}(d-1)$-bounces of field theory 11] because a massless vortex does not 'bounce' back in the potential.

[11] H. Kao, K. Lee, Phys. Rev. D 52, 6050 (1995)

[12] J. C. Davis et al., Phys. Rev. Lett. 69, 323 (1992)

[13] G. G. Ihas et al., Phys. Rev. Lett. 69, 327 (1992)

[14] E. A. Kuznetsov, J. Juul Rasmussen, JETP Lett. 62, 105 (1995); C. Josserand, Y. Pomeau, Europhys. Lett. 30 (1), $43(1995)$

[15] O. Avenel, G. G. Ihas, E. Varoquaux, J. Low Temp. Phys. 93, 1031 (1993) 